\title{
Performance Optimization of Vision Apps on Mobile Application Processor
}

\author{
Kwang-Ting (Tim) Cheng, Xin Yang and Yi-Chu Wang \\ Dept. of Electrical and Computer Engineering, University of California, Santa Barbara, CA, 93106, USA \\ timcheng@ece.ucsb.edu, xinyang@umail.ucsb.edu, yichuwang@umail.ucsb.edu
}

\begin{abstract}
Optimizing performance of compute-intensive vision apps running on mobile application processor (AP) is critical to satisfactory experience for smartphone and tablet users. Most existing vision algorithms were primarily designed and implemented for desktop and server platforms. Porting them to a mobile platform without adapting the algorithms to account for the platform's limitations would cause serious algorithmhardware mismatches, yielding unnecessary runtime degradation. Modern mobile AP, which integrates multi-core CPUs, GPUs and other special-purpose accelerators, offers multiple options of porting vision apps to various computing cores. To develop an optimized implementation for a vision app, it is necessary to understand the potential mismatches for better algorithm adaptation and optimized mapping of the algorithm to a handheld platform. In this paper, we identify mismatches and propose adaptation guidelines for three different porting strategies: porting an algorithm to 1) a mobile CPU, 2) a mobile GPU, and 3) mobile heterogeneous multi-cores (mobile CPUs+GPUs). For each strategy, we illustrate the adaptation/porting guidelines using an exemplar vision task. Experimental results demonstrate that with proper adaptation following the proposed guidelines, we could achieve a significant speedup with little accuracy drop.
\end{abstract}

Keywords-mobile application processor; algorithm adaption; GPU acceleration; heterogeneous parallel computing

\section{INTRODUCTION}

Computer vision, which brings together multiple fields such as image processing and artificial intelligence, has derived many vision apps permeating into our daily life. With the pervasive presence of high quality cameras on mobile devices, combined with the increasing computing capabilities of mobile $\mathrm{AP}$, we are witnessing a rising deployment of vision apps on mobile devices, e.g. mobile augmented reality [5], mobile photo management [8], etc. However, most existing vision apps are compute-/memory-intensive, and thus running such demanding apps without any optimizations on mobile devices is usually too slow to meet a satisfactory user experience.

While many vision algorithms have been optimized [2] [3], most existing optimizations are based on general computing platforms, such as laptops, desktops and servers, not based on mobile handheld devices. The AP of mobile handheld devices differ from that of general computing systems in several aspects, e.g. different processor micro-architecture, system memory bus architecture, and memory bandwidth. As a result, porting desktop-oriented optimizations to mobile platforms without considering mobile hardware limitations would lead to mismatches between algorithms and mobile hardware, yielding

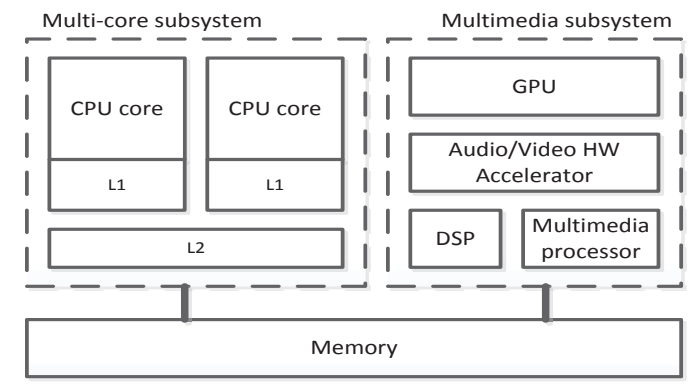

Figure 1: Common system architecture of a mobile AP which integrates a multi-core CPU, GPU, DSP and other accelerators in a single chip.

unnecessary slow-down. Identifying the mismatches and adapting algorithms accordingly are critical for performance optimization of vision apps on mobile APs, which are yet to be well studied.

Modern mobile AP integrates various computing cores, including CPUs, GPUs and other application-specific accelerators, in a single chip (Figure 1). It provides developers multiple ways for application-to-platform mapping, i.e. porting it to a single core (e.g. CPU or GPU) or heterogeneous multiple cores (e.g. CPUs+GPUs). While it is desirable to have a best mapping strategy for general vision tasks, it is inevitably taskdependent and platform-dependent due to distinct computational characteristics of different computing cores when performing different vision tasks. A better understanding of the potential mismatches in each mapping strategy allows us to well explore the true benefits of each strategy, facilitating a fair comparison among different optimized mapping strategies.

In this paper, we focus on optimizing the performance for three common porting strategies: porting an algorithm to 1) a mobile CPU, 2) a mobile GPU, and 3) heterogeneous mobile cores (i.e. mobile CPUs+GPUs). For each strategy, we identify algorithm-hardware mismatches and provide corresponding adaptation guidelines. For each strategy, we also use an exemplar vision task to demonstrate that tuning the algorithms according to our adaptation guidelines can significantly reduce the overhead caused by mismatches, and in turn, reducing the runtime. Meanwhile, algorithm adaptation for performance optimization causes very little compromise in the accuracy.

\section{AlgORITHM AdAPTION TO MOBILE CPU}

As a major computing unit in mobile AP, modern mobile CPUs are designed with much more than pure speed in mind. Priority is often given to factors which address requirements of 
a mobile operating environment such as low power consumption and small form factor size. Based on these considerations, mobile CPUs usually feature less advanced hardware implementations and smaller memory capacity than the server and desktop CPUs. These limitations may lead to algorithm-hardware mismatches when porting desktop-oriented apps to a mobile CPU. In this section, we identify two mismatches and provide corresponding adaptation methods.

\section{A. Mismatches between Computations and Mobile CPU}

Many vision tasks have two common computation characteristics: 1) iteratively accessing image data using a sliding window, and 2) involving a large amount of datadependent branch operations. For instance, most, if not all, point detection algorithms apply box filters to every image pixel using a sliding-widow-based fashion (see Figure 2 (a)). Many decision tree-based algorithms involve extensive judgments to decide successive steps based on current results. These two types of computations, which won't cause problems on most desktop CPUs, may cause overheads on a commodity mobile CPU:

- Mismatch between sliding-window-based data access pattern and small cache size of a mobile CPU. As a 2D image array is stored in a row-based fashion in memory (cache and DRAM), not in a window-based fashion; pixels in a single sliding window reside in multiple memory rows. The data cache size of a mobile CPU, typically $32 \mathrm{~KB}$ for today's devices, might be too small to cache all memory rows for pixels involved in one sliding window, leading to cache misses and cache line replacements and, in turn, incurring expensive memory access.

- Mismatch between data-dependent branches and high pipeline hazard penalty of the mobile CPU. Data-dependent branches may cause pipeline hazards penalty. Modern mobile processors usually use a deep pipeline to increase the CPI, which increase the penalty of each mis-branch-prediction. Moreover, for mobile CPUs, the branch predictor hardware and other hazard solutions (e.g. out-of-order execution), while improving for each new generation, are still not as sophisticated as desktop CPUs. Consequently, a large amount of data-dependent branches would incur high pipeline hazard penalties, yielding significant runtime degradation.

- Adaptation Guidelines. When porting vision algorithms to a mobile CPU, it is necessary to estimate the overhead caused by these two mismatches. If lots of cache misses or branches exist, it is better to adapt the algorithms to fit more demanded data into the cache (e.g. by re-arranging the data) or reduce branch operations as many as possible (e.g. by implementation/algorithm replacement) to reduce overhead. Next, we use SURF point detection as an example to demonstrate the adaptation process and benefits.

\section{B. Adapting SURF to Mobile CPU}

\section{(1) Review of SURF Point Detection}

SURF (Speed-Up Robust Feature) [1] point detection is a fundamental function in many vision tasks. It consists of two steps: 1) localizing a set of salient points, and 2) computing a dominant orientation for each detection point. In the first step, a SURF detector accesses an integral image [1] using a sliding window of successively larger size to identify salient points over scales (Figure 2(a)). In the second step, a SURF detector (a) Locality based Original SURF
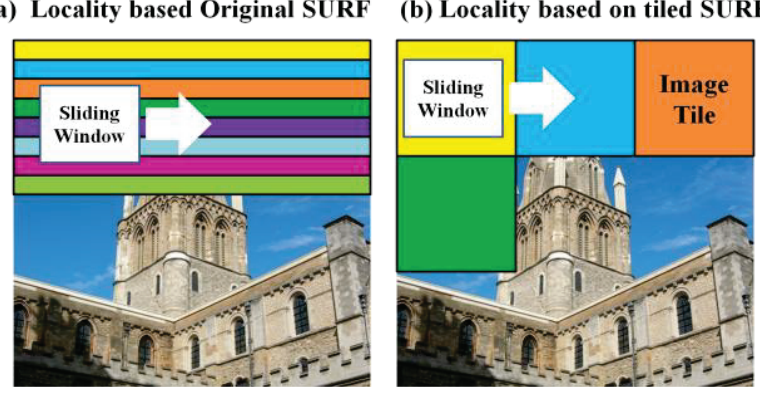

Figure 2. Illustration of data locality and access pattern in (a) the original SURF detector, and (b) the tiled SURF. Each color represents data stored in a unique DRAM row. In the original SURF, a sliding window needs to access multiple DRAM rows, leading to frequent cache misses, while in tiled SURF, all required data within a sliding window can be cached.

identifies a dominant orientation for each salient point based on gradient histograms. In particular, a SURF detector quantizes the entire orientation space into histogram bins using overlapping windows. Then it maps every pixel around a salient point to corresponding bins via a set of branch operations according to the pixel' gradient orientation. After processing all neighboring pixels around a salient point, a bin with most votes is selected to compute a dominant orientation. As the total number of pixels involved in this analysis is huge, the entire process involves an enormous amount of datadependent branch operations which are not easily predicted.

\section{(2) Adaptation Methods}

To address the problem caused by the mismatch between data access pattern of SURF and small cache size of a mobile CPU, we proposed tiled SURF which divides an image into tiles (see Figure 2 (b)) and performs point detection for each tile individually to exploit local spatial coherences and reduce external memory traffic. To avoid pipeline hazards penalties, we propose two solutions to remove data-dependent branch operations. The first solution is to use an alternative implementation, i.e. instead of using "If-then-Else" expressions we use a lookup table to store the correlations between each orientation and the corresponding histogram bins. This solution does not change the functionality and other computations, but trades memory for speed. The second solution is to replace the original gradient histogram method with a branching-free orientation operator based on gradient moments (GMoments) [3]. The gradient-moment-based method may slightly degrade the robustness of a SURF detector, but can greatly improve the speed on mobile platforms [4].

\section{(3) Results}

We compare the time cost and the Phone-to- $P C$ ratio between the original SURF and adapted SURF in Table 3 and 4 respectively. The Phone-to- $P C$ ratio is the runtime of a program running on a mobile $\mathrm{CPU}$ divided by that on a desktop CPU, which reflects the speed gap between them.

$$
\text { Phone }- \text { to }-P C \text { ratio }=\frac{\text { runtime on mobile CPU }}{\text { runtime on desktop CPU }}
$$

We perform experiments on three mobile devices: a Motorola Droid which features an ARM Cortex-A8 processor, an HTC 
Table 1. Time Cost Comparison on Three Mobile Platforms

\begin{tabular}{|c|c|c|c|}
\hline Time (ms) & Droid & $\begin{array}{c}\text { Thunder } \\
\text { bolt }\end{array}$ & Xoom1 \\
\hline U-SURF & 1310 & 525 & 461 \\
U-SURF Tiling & 930 & 356 & 243 \\
\hline O-SURF & 7700 & 2495 & 2156 \\
O-SURF Lookup-Table & 2714 & 1043 & 946 \\
O-SURF GMoment & 1516 & 613 & 519 \\
\hline O-SURF Tiling+GMoment & 1053 & 404 & 269 \\
\hline
\end{tabular}

Table 2. Speed Ratio Comparison on Three Mobile Platforms

\begin{tabular}{|c|c|c|c|}
\hline Phone-to-PC Ratio (x) & Droid & $\begin{array}{c}\text { Thunder } \\
\text { bolt }\end{array}$ & Xoom1 \\
\hline U-SURF & 20 & 8 & 7 \\
U-SURF Tiling & 14 & 7 & 4 \\
\hline O-SURF & 54 & 17 & 15 \\
O-SURF Lookup-Table & 18 & 7 & 6 \\
O-SURF GMoment & 19 & 8 & 7 \\
\hline O-SURF Tiling+GMoment & 13 & 7 & 3 \\
\hline
\end{tabular}

Thunderbolt which uses a Scorpion processor, and a Motorola Xoom1 which uses a dual-core ARM Cortex-A9 processor.

The first two rows of Tables 1 and 2 compare the time cost and Phone-to-PC ratio of upright SURF (U-SURF) without and with tiling. As expected, tiling can greatly reduce time cost by $29 \% \sim 47 \%$. At the same time, it reduces the Phoneto- $P C$ ratio by $12.5 \% \sim 42.9 \%$ on the three devices. The reduction in Phone-to- $P C$ ratio indicates that the mismatch between data access pattern and a small cache size of a mobile CPU causes more severe runtime degradation on mobile CPUs than desktop CPUs. So alleviating this problem is critical for performance optimization when porting algorithms to a mobile CPU. The $3^{\text {th }} \sim 5^{\text {th }}$ rows of Tables 1 and 2 compare the results of oriented SURF (O-SURF) with branch operations, O-SURF using a lookup table and using GMoment, respectively. Results show that using lookup table or using GMoment method can greatly reduce the overall runtime and the Phoneto- $P C$ ratio on three platforms. The reduction in Phone-to- $P C$ ratio further confirms that branch hazard penalty has a much greater runtime impact on a mobile CPU than on a desktop CPU. Choosing proper implementations or algorithms to avoid such penalties is critical for a mobile task. The last rows of Tables 3 and 4 show the results with the application of both two adaptations to O-SURF: comparing to original SURF, the two adaptations can reduce the runtime on mobile platforms by $6 \mathrm{X} \sim 8 \mathrm{X}$.

\section{ALGORITHM ADAPTION TO MOBILE GPU}

Exploring the use of GPU as a general-purpose coprocessor to accelerate compute-intensive applications in desktop and server systems, known as GPGPU, has been an active research subject for a decade. Many parallelizable, GPU friendly algorithms have been proposed to leverage the computing power of GPU's massive parallel computing architecture. Mobile GPU, however, has very different characteristics compared to desktop GPUs, i.e. a mobile GPU usually has fewer cores, smaller graphics memory, lower GPU bus bandwidth, and variant architecture when compared to a

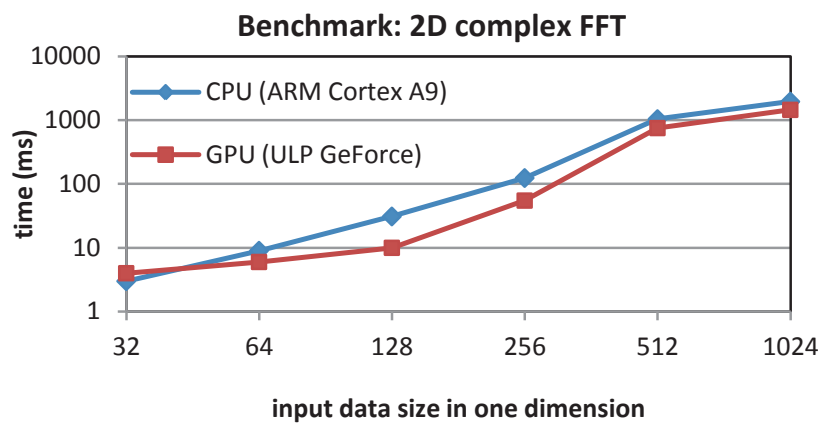

Figure 3. Performance comparison of mobile CPU and GPU

desktop GPU. It is desirable to characterize the computing capability of a mobile CPU-GPU platform and to identify the condition that offloading tasks to a GPU leads to a better performance than computing the tasks in a CPU.

\section{A. Mismatches between Data Size and Mobile GPU}

There are runtime overheads, including the initialization of GPU and data transfer between CPU and GPU, associated with the usage of GPU. Algorithm-hardware mismatches may intensify these overheads, and in turn affect the decision of using GPU for acceleration or not

- Mismatch between small data size and GPU initialization overhead. For small input data size, the initialization overhead is larger than the reduced execution time by GPU acceleration, yielding an inferior performance of using GPU to using CPU.

- Mismatch between large data size and small cache size of $\boldsymbol{G P U}$. For a large-size data that cannot fit into the cache of a GPU, expensive off-chip memory transactions would be required. As GPU usually has a smaller cache than CPU, once the overhead caused by off-chip memory accesses exceeds the time reduced by GPU acceleration, GPU provides an inferior performance to $\mathrm{CPU}$.

\section{B. Performance Characterization of Mobile GPU}

We use FFT (Fast Fourier Transform) as a benchmark to characterize the computing capability and limitation of a mobile GPU and make comparisons to a mobile CPU. FFT is a basic operation in many signal processing algorithms. It is also a widely used benchmark to compare performance of various architectures.

To utilize a mobile GPU as a general-purpose accelerator, programmers have to map the algorithms to the graphics operations since the GPGPU programming framework, such as CUDA and OpenCL is not supported in mobile devices. OpenGL ES 2.0 [6] is the primary graphics programming interface for handheld and embedded devices with a programmable GPU. The Discrete Fourier Transform (DFT) of an 1D function $x(n)$ is given by $X(k)=\sum_{n=0}^{N-1} x(n) e^{-2 \pi n k / N}$, where $0 \leq k \leq N-1$. The Cooley-Tukey FFT algorithm is a widely used method to compute DFT: it recursively decomposes Fourier Transform into smaller size Fourier Transforms. In each recursive stage, every two input samples is grouped and performed $y_{0}=x_{0}+w^{0} * x_{1}$ and $y_{0}=x_{0}-$ $w^{0} * x_{1}$, where $x_{0}$, and $x_{1}$ are input samples of a stage and $y_{0}$, and $y_{1}$ are the output samples of this stage. It is also called butterfly computation. The vertex shader of GPU is programed 


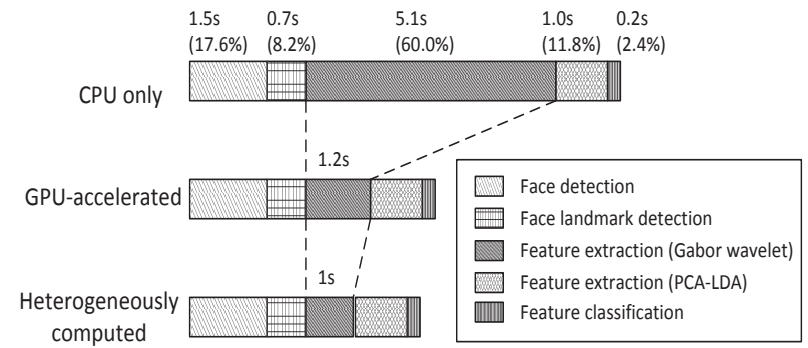

Figure 4. Comparison of face recognition system running on Tegra platform with three different computing strategies.

to render a quad with the size equal to the length of sample. The fragment shader of GPU is programmed to compute the butterfly computation. Each stage requires a texture of size $N \times 1$ to store the pre-computed coefficients (i.e. $\pm w^{0}$ ). A total of $\log _{2} N$ stages are required to complete the computation for $\mathrm{N}$ samples. This recursive processing is implemented by multiple rendering passes, with a floating point framebuffer object (FBO) to store the intermediate rendering results.

Figure 3 compares the performance of two computing cores in Tegra 2 SoC. The integrated mobile CPU is ARM CortexA9, and the integrated mobile GPU is ultra-low power (ULP) GeForce which has 8 unified vertex and fragment shaders. From Figure 3 we can see that there is a preferred region to accelerate computation using mobile GPU. For this platform configuration, the highest speedup is achieved by data size of $128 \times 128$ : the GPU is $3 \mathrm{X}$ faster than the CPU.

\section{GPU-Accelerated Face Recognition}

We demonstrate the benefits of mobile GPU acceleration for mobile face recognition. The task of face recognition is to identify faces via three steps: 1) localizing face positions, 2) representing the face image using feature vectors, and 3) comparing to pre-defined face feature database. A robust face feature extraction is the key to high accuracy of face recognition but is also the most time consuming part of recognition pipeline. A design instance based on Gabor wavelet feature [7] takes $8.5 \mathrm{~s}$ to detect and recognize a person when running on Nvidia's Tegra2 AP. The kernel computation of Gabor wavelet is $80128 \times 128$ FFT. By offloading this computation to Tegra2's GPU, the Gabor face feature extraction task can be reduced from $5.1 \mathrm{~s}$ to $1.2 \mathrm{~s}$, which represents a $4.25 \mathrm{X}$ speedup in comparison with the $\mathrm{CPU}$ implementation. The first two bars in Figure 4 details the runtime breakdown of face recognition when computing using CPU-only vs. with GPU-acceleration.

\section{CONCURRENT COMPUTING USING BOTH CPU AND GPU}

Mobile users expect applications to be responsive, not like many of desktop or server applications are batch computations. This is especially true for interactive vision applications. An effective strategy to enhance mobile application's user experience is to utilize all the computing cores concurrently to complete a task. A potential pitfall in performance of this strategy is the contention of common system resources among computing cores [8].

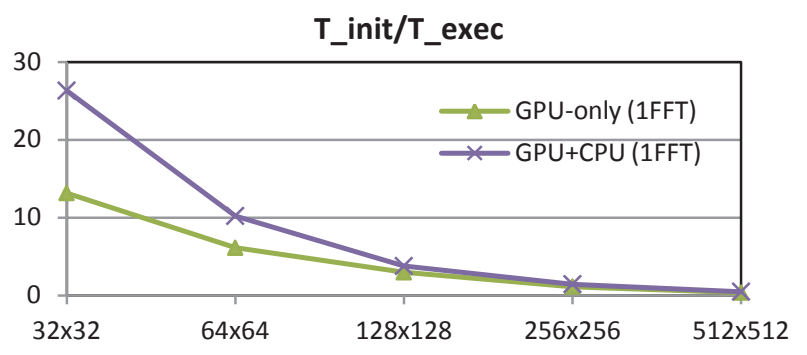

Figure 5. Initialization cost presented as the time ratio of initialization and execution. When CPU core computes its own task in the background, the initialization cost almost doubles.

\section{A. Performance Characterization of Heterogeneous Cores}

There are two types of resource shared among cores in mobile AP: CPU's computing resource and system bus and memory. The GPU core and other accelerators are designed as co-processors of CPU and need to be initialized by CPU before performing computing task. For example, a GPU initialization phase includes allocating rendering buffer, compiling shader program, and configuring relative rendering settings. These processes occupies significant amount of CPU utilization. If CPU is also executing its own computing task at the same time period, the time of the initialization phase will be doubled. We have revealed in previous section that GPU initialization time is significant in overall runtime when process small size FFT. This initialization time becomes more significant in heterogeneous concurrent computing mode, i.e. when both CPU and GPU are executing tasks. To alleviate this overhead, the computation task should be intensive enough, which could be either with larger data size or more computing subtasks. In Figure 5, we show the initialization cost as a ratio of initialization time and execution time. For example, the initialization cost is equivalent to "computing $1332 \times 32$ FFT" in GPU-acceleration mode, and "computing $2632 \times 32$ FFT" in concurrent mode. If we have a computing task of 26 FFT, incorporating $\mathrm{CPU}$ core in addition to GPU will not be beneficial for reducing the runtime.

After the initialization stage, the coprocessor GPU can execute the given computing task on its own. In this execution phase, the time to compute a task also is impacted by other cores running tasks in background. In the execution phase, the ratio of GPU runtime with and without CPU executing task in the background range from 1.2 (for $32 \times 32$ FFT) to 1.4 (for $512 \times 512$ FFT). This concurrent overhead is less significant compare to the initialization stage.

\section{B. Accelerating Face Recognition on Heterogeneous Cores}

Finally, we apply this heterogeneous concurrent computing strategy in the face recognition application. Extracting feature of a face requires computation of 80 FFT. We partition these tasks into two based on the relative performance ratio of the CPU and GPU cores and the concurrent overhead. Result is shown in the last bar of Figure 4 . When processing one face, the speedup is $5 \mathrm{X}$ comparing to using CPU alone, and is $1.2 \mathrm{X}$ comparing to offloading to GPU. More speedup can be achieved when recognizing more faces, as the GPU initialization cost is diluted. 


\section{CONCLUSION}

In this paper, we study three application-to-platform mapping strategies. For each strategy, we identify algorithmhardware mismatches when porting desktop-oriented vision algorithms to a mobile application processor. We provide algorithm adaptation guidelines to alleviate unnecessary performance degradation caused by these mismatches and demonstrate the performance gain of employing the proposed guidelines to exemplar vision tasks running on mobile APs.

\section{REFERENCE}

[1] Bay, H., Ess, A., Tuytelaars, T. and Gool, L.V.. SURF: Speeded-up Robust Features. In Proc. of ECCV'06.

[2] Terriberry, T. B., French, L. M., and Helmsen, J.. GPU Accelerating Speeded-Up Robust Features. In Proc. of 3DPVT'08.
[3] Chen, W.C., Xiong, Y. G., Gao, J., Gelfand, N. and Grzeszczuk, R.. Efficient Extraction of Robust Image Features on Mobile Devices. In Proc. of ISMAR'07.

[4] Yang, X. and Cheng, K. T.. Accelerating SURF Detector on Mobile Devices. In Proc. of ACM MM'12.

[5] Yang, X. and Cheng, K. T.. LDB: An Ultra-Fast Feature for Scalable Augmented Reality on Mobile Devices. In Proc. of ISMAR'12.

[6] Khronos Group, OpenGL ES 2.0 Specification, http://www.khronos.org/opengles.

[7] Su, Y., Shan, S., Chen X. and Gao, W., "Hierarchical Ensemble of Global and Local Classifiers for Face Recognition," IEEE Transactions on Image Processing, vol.18, no.8, pp.1885-1896, 2009.

[8] Wang, Y. C. and Cheng, K. T.. "Energy and performance Characterization of Mobile Heterogeneous Computing". In Proc. of IEEE SiPS'12. 